\section{Moda, estilo y ciclo de vida de los productos de la industria textil}

Aníbal Bur *

Resumen: La moda refleja el gusto de una época, los valores y las opiniones dominantes. Cuando las personas piensan en la moda, generalmente, imaginan un mundo de fantasía conformado por diseñadores, modelos y prendas atractivas. En realidad, la moda no se refiere solamente a la indumentaria, sino que se aplica a cualquier producto o servicio de consumo masivo como, por ejemplo, automóviles, música, mobiliario, revistas, destinos turísticos, etc.

Si consideramos la moda desde el punto de vista de la indumentaria, encontramos que tiene una dimensión sociocultural y, además, una dimensión económica. En relación a esta última, podemos señalar que el marketing de la moda tiene como funciones determinar qué productos se van a vender, a quiénes, a qué precios, cómo se van a comunicar y cuáles serán sus canales de distribución.

Frecuentemente, los términos moda y estilo se confunden. La moda se refiere a todo producto que se convierte en popular durante un período de tiempo y en un determinado lugar. En cuanto al estilo, éste se refiere a las características y los detalles que diferencian unos productos de otros. Dentro de un mismo estilo puede haber muchas variaciones en su creación: estamos en presencia de los diseños. Siempre hay muchos más diseños que estilos. Cuando un estilo se convierte en popular se transforma en moda.

A los productos de moda del rubro indumentaria, al igual que cualquier producto, se les puede aplicar el modelo de ciclo de vida. Este modelo supone que los productos que se introducen a los mercados competitivos atraviesan un ciclo predecible en el tiempo. Cada etapa recibe un nombre específico: introducción, crecimiento, madurez y declinación. Conocer la etapa del ciclo en la que se encuentra el producto de moda permite a los especialistas de marketing, diseñar la estrategia más eficaz para comercializarlo, aspecto especialmente relevante en los mercados altamente competitivos en los que la industria textil debe actuar.

Palabras claves: ciclo de vida - diseño - estilo - indumentaria - marketing - moda - modelo novedades.

[Resúmenes en inglés y portugués en las páginas 153-154]

${ }^{(*)}$ Doctor de la Universidad de Buenos Aires. Psicólogo Social graduado en la Primera Escuela Privada de Psicología Social y especializado en Psicología Social de las Organizaciones. Se graduó como Profesor Universitario en la Universidad del Museo Social Argentino (UMSA) y se especializó en Ciencias Empresariales. Capacitador y consultor en management y marketing en empresas de productos y/o servicios nacionales e internacionales. 
El Diccionario de la Real Academia Española define a la moda (del fr. mode) como "uso, modo o costumbre que está en boga durante algún tiempo, o en determinado país, con especialidad en los trajes, telas y adornos, principalmente los recién introducidos". De acuerdo a esta definición, la moda no se refiere exclusivamente a la indumentaria y sus accesorios. El concepto de moda, entonces, se aplica a cualquier producto o servicio de consumo masivo como, por ejemplo, automóviles, música, mobiliario, revistas o destinos turísticos. Sin embargo, las personas cuando piensan en la moda, por lo general, imaginan un mundo de fantasía conformado por diseñadores, modelos y ropa atractiva.

La moda refleja el gusto de una época, los valores y las opiniones dominantes. Es decir, es el reflejo y la expresión estética de una sociedad. Por lo tanto, permite identificar cómo la gente piensa en ese momento. En cada época, la moda es impuesta no sólo por personalidades como pueden ser los artistas, los políticos, etc., sino que también, se crea a partir de los valores y actitudes sociales imperantes en ese momento. Esto es así porque la moda es un comunicador de identidad. Por ejemplo, cuando un grupo se identifica con la vida natural, su indumentaria refleja claramente su pensamiento. La religión, los niveles de educación de las personas, la posición social crean necesidades diferentes que se reflejan en la indumentaria. Por otra parte, las expresiones a favor o en contra del consumismo que hubo en diferentes épocas, se manifestaron a través de la vestimenta y el arreglo personal.

Durante el siglo XX la mujer alcanzó libertades sociales, políticas y económicas. Esta nueva forma de vida requirió ropa, peinados y accesorios más cómodos y libres acordes con esa necesidad de mayor actividad. La ropa de ama de casa ya no se ajustaba a las necesidades de la mujer que trabajaba fuera del hogar. En la actualidad, las mujeres están más tiempo fuera de su casa y desarrollan actividades diversas por lo cual necesitan ropa que se adapte a todas las funciones (trabajo, estudio y salidas nocturnas); la ropa pret-à-porter es la solución a estas demandas.

La historia, el clima, la geografía del propio hábitat, las nuevas tendencias, como por ejemplo el cuidado mayor del cuerpo en los '70 y los ' 80 , generan nuevas necesidades que se reflejan en la indumentaria.

La moda también responde a los cambios en el estilo de vida de la sociedad. Por ejemplo en lo que respecta a la moda femenina, en los ' 40 se impuso la pollera amplia y la chaqueta entallada que marcaba la cintura; en los ' 50 se impusieron los pantalones para estar cómoda y en movimiento; en los '60 Mary Quant impuso la minifalda y André Courrèges el corte cuadrado con chaquetas cortas; en los '70 se impone como estética la mujer estilizada y la ropa suaviza sus formas con otras texturas de las telas; en los ' 80 aparecen las grandes hombreras y las polleras tubo; en los '90 la tendencia fue mostrar más el cuerpo de la mujer.

Por otra parte, la moda también se crea a partir del desarrollo tecnológico, por ejemplo, la creación de nuevas fibras (wash and weare, lycra, etc.) tuvo su impacto en la fabricación de prendas de vestir. También, las nuevas tecnologías permitieron otorgar flexibilidad, tersura, maleabilidad, estética y belleza a distintos materiales como, por ejemplo, cueros y plásticos lo que impactó en la fabricación de indumentaria.

Desde el punto de vista sociológico, Piñuel Raigada (1992) expresa que la no aceptación de las innovaciones que propone la moda es considerada una señal de marginación, mientras que la participación en las mismas se premia con el reconocimiento social. Este autor considera que el desencadenante esencial de la creación y desarrollo de las modas es determinado por signos de poder como, por ejemplo, la distinción social. Además, agrega que "el deseo de imitar las 
formas de vida de los estamentos de poder, se convierte en la garantía de la eficacia para la implantación o seguimiento de cualquier moda". De acuerdo a este enfoque, la moda fluiría desde la punta de la pirámide social hacia abajo, circulando en forma horizontal (entre un mismo grupo social) o transversal (entre grupos no vinculados). Otros especialistas consideran que, en la actualidad, es menor la imitación de "clases de aspiración" y que la forma de "filtrado" tradicional se ha dado vuelta y las modas empiezan a fluir de abajo hacia arriba. Además, podemos agregar que existen grupos que forman fuertes subculturas propias o movimientos propios de una época (floggers, emos, rappers, cumbieros, skaters, punks, neohippies, góticos, rollingas, etc.) que crean sus propias modas.

De acuerdo a lo señalado anteriormente, podemos considerar tres teorías que intentan explicar como se expande e impone una moda:

1. Teoría del escurrido: la moda se escurre de arriba hacia abajo. Por ejemplo, en el pasado los reyes imponían la moda que copiaban los nobles y las clases acaudaladas. Las clases más bajas quedaban excluidas de este proceso.

2. Teoría de la navegación: se desarrolla con el progreso del siglo XX donde se reduce la tendencia a copiar la indumentaria. En esta época se consideraba que la ropa debía ser funcional y estaba destinada a acompañar el rol que cada uno cumplía en la sociedad. Esta teoría sostiene que la moda se expande horizontalmente en cada grupo y, también, puede circular entre grupos no vinculados entre sí. Es decir, algunas modas permanecerán dentro del grupo que las adoptó, mientras que otras fluirán de un grupo a otro y se usarán masivamente. Cada subcultura o grupo, tiene sus propios líderes y la intención de sus integrantes sería identificarse con el grupo a través de la indumentaria como forma de aceptación por los iguales.

3. Teoría del cambio de abajo hacia arriba: diversos especialistas sostienen que la forma de filtrado tradicional se ha dado vuelta y ahora las modas pasan de abajo hacia arriba. Por ejemplo, los jeans, las zapatillas, las alpargatas, la ropa safari o las camperas de cuero, típicas prendas usadas actualmente por todos los estratos sociales comenzaron a usarse en clases bajas y, posteriormente, las adoptaron consumidores de mayores recursos. Sproles (1985) considera que casi cualquier individuo creativo e innovador puede imponer una tendencia si esta es acorde al clima social y con los estilos de vida imperantes en esa época.

Continuando con el enfoque sociológico, podemos señalar que la moda es un acto de comunicación. Constituye una forma de comunicación no verbal, por la que las personas expresan su identidad y pertenencia a la sociedad. Sin embargo, Saulquin (1998), si bien opina que la indumentaria es un medio de comunicación efectivo, considera que hay que preguntarse si la información que transmite se ajusta a la realidad o, por el contrario, la oculta. Esto último se evidencia cuando las personas, a través de la vestimenta, quieren aparentar la pertenencia a una determinada clase social en la que, en realidad, no están incluidos.

Los especialistas de la moda intentan descifrar los patrones de conducta que mueven a los consumidores a seguir una moda. Este fenómeno comienza con pocos usuarios y rápidamente se expande a muchos consumidores. Esto puede deberse, entre otras razones, a que siguen a un líder de opinión. Los psicólogos sociales señalan que la actitud de seguir al líder parece responder 
a un deseo individual de obtención de status a través de la elección de cierta ropa igual a las que usan personas o grupos admirados. Esta identificación es tomada como una manera de acortar las distancias entre los grupos sociales. La idea es que "si me visto como ellos, me voy a parecer". Los expertos afirman que la moda provee a las personas de status y les otorga seguridad al favorecer la adaptación. En este sentido, la moda permite satisfacer estas dos necesidades humanas. En cuanto al comportamiento del consumidor podemos observar distintas posiciones, desde la inseguridad de los copiadores a la audacia de los que visten una prenda que nadie ha usado antes. Este último comportamiento puede deberse al coraje del consumidor o a razones de autopromoción, por ejemplo, como lo hacen los artistas a sugerencias de sus productores.

En la conducta de los consumidores hay que resaltar la influencia de las grandes casas de alta moda y de los diseñadores. Sus creaciones, por lo general, crean tendencias y los demás las siguen. Los diseñadores entienden que las mujeres buscan cambio e innovación y, por lo tanto, pretenden satisfacerlas a partir de sus nuevas creaciones.

La palabra del diseñador, del productor de modas o del vendedor ya no alcanzan para imponer una moda. Como señalamos anteriormente, las modas se desarrollan por diversos motivos: cambios sociales, cambios en las actitudes y las preferencia, desarrollo tecnológico, cambios en el medio ambiente o grandes acontecimientos mundiales. Es importante considerar que todo depende del momento, del país o lugar específico y del grupo o población que esté involucrada en ese cambio.

Distintos autores trataron de explicar el fenómeno de los cambios en la moda en la sociedad a partir de diferentes puntos de vista (Barthes, 1978; König, 1972; Saulquin 1998). Al respecto, Sproles y Burns (1994), establecieron dos categorías para explicar los cambios de la moda. La primera categoría parte del supuesto que la industria de la moda es la que provoca el inicio de los cambios creando nuevas tendencias. En este sentido, las casas de moda europeas ejercen una acción intensa y poderosa sobre los patrones de la moda. En realidad, construyen preferencias de la industria y, por lo tanto, en los consumidores. Hay corrientes que reaccionan frente a esta influencia, invitando a los consumidores a resistir las modas impuestas artificialmente y a consumir lo que realmente satisface sus necesidades. La segunda categoría considera que los consumidores inician el cambio. En este caso, los mismos enfrentan un espectro muy amplio de productos y estilos distintos y determinan cuáles serán usados ampliamente y cuáles desaparecerán sin que nadie los adopte.

En la moda, lo único que permanece constante es el cambio. Esto es así porque la moda es un símbolo de la realidad y ésta cambia. Además, la moda se corresponde a redes ocultas e intrincadas que operan sobre la realidad (Beaton, 1990).

El experto en moda sabe que lo importante es el cambio, sin embargo, la gente rechaza los cambios bruscos. El diseñador desarrolla nuevas ideas pero se encamina lentamente hacia ellas a medida que construye el gusto del consumidor en forma gradual y contínua. Hay excepciones, como por ejemplo Christian Dior quien, después de la segunda Guerra Mundial, introduce el new look concepto de mujer liberada, participativa y femenina.

La naturaleza evolutiva de los cambios de la moda es de vital importancia para los creadores, ya que al captarla obtienen fundamentos para pronosticar e identificar la moda que viene. La mente del creador tiene presente la moda actual y trabaja en todas las direcciones que existen mientras va concibiendo y diseñando sus modelos. Una prenda muy aceptada en la temporada 
de invierno da imperceptibles señales en sus detalles los que son tomados y recreados por los diseñadores para la temporada de verano.

Cuando una tendencia llega al extremo, comienza el cambio hacia una dirección opuesta. Por ejemplo, cuando la pollera no puede ser más corta vuelve la pollera larga. Al respecto, Paul Poiret (1930) señala que todas las modas terminan en exceso. Para los creadores de la moda, es importante reconocer y analizar estos cambios que definen la tendencia y que deben estar un paso adelante que el consumidor, identificando estas fluctuaciones para ver quiénes proponen el cambio. Cabe señalar que, estas señales o indicadores de cambio son imperceptibles y requieren un entrenamiento específico para detectarlas. En este sentido la intuición no es lo más importante sino que lo que cuenta es la experiencia.

Desde el punto de vista del negocio de la moda, es necesario definir claramente algunos conceptos. Grünfeld (1997) señala que, frecuentemente, se confunden los términos moda y estilo. Aclara que la moda se refiere a todo producto que se convierte en popular debido a su aceptación social, durante un período de tiempo y en un determinado lugar. En otras palabras, la moda es todo producto que es aceptado y adoptado socialmente y que, a la vez, se destaca de otros de la misma categoría, tanto en preferencias como en la decisión de compra por parte de los consumidores. En cuanto al estilo, éste constituye una tipología del producto. Es decir, se refiere a las características y los detalles que lo diferencian de otros, por ejemplo, en indumentaria podríamos señalar los sacos con hombreras, o las polleras tubulares.

En el rubro indumentaria, dentro de un mismo estilo puede haber muchas variaciones en su creación las que se denominan diseños. Por lo tanto, siempre habrá muchos más diseños que estilos. A partir de lo señalado anteriormente, cuando un estilo se convierte en popular se transforma en moda. Por otra parte, un estilo puede volver repetidas veces, en cambio la moda tiene un carácter pasajero. La moda, en tanto símbolo de la realidad, cambia en forma continua. Cabe señalar que cuando vuelve un estilo (revival), lo hace metamorfoseado, es decir, modificado. Así, un estilo clásico puede perdurar un largo período de tiempo. En el caso de la indumentaria, un estilo clásico significa sobrevivir a varias temporadas, por ejemplo, las remeras abotonadas tipo Lacoste. En cambio, las novedades son tendencias pasajeras que no tienen impacto en las modas futuras. Son estilos que se imponen repentinamente en forma total por un tiempo breve y luego desaparecen sin rastros.

La prenda única que posee ciertas características pertenecientes a un determinado estilo o que crea un estilo nuevo, se denomina modelo. Los modelos se presentan en las colecciones de alta moda y, por lo tanto, se producen y venden en pequeñas cantidades hasta que dejan de ser una novedad. Si uno de estos modelos interesa a un público más amplio, se copia, se fabrica en grandes cantidades y se vende a los consumidores a precios más bajos. Por supuesto, los clientes que compraron los modelos originales ya no los usan y buscan nuevos modelos que crean los diseñadores.

La moda, desde el punto de vista de la indumentaria, es un sistema particular, tanto por su funcionamiento como por su mercado. Se trata de un mercado cuya oferta debe responder a una demanda multiforme y en continuo cambio. En este sentido, la moda tiene una dimensión sociocultural y, además, una dimensión económica.

Para entender la industria de la moda es necesario considerar cómo se crea y gestiona una marca, cuáles son las políticas comerciales y de fidelización de clientes y qué actitud deben tomar las 
empresas de la moda para competir en un mercado globalizado como el que plantea internet. La moda es imagen y los productos de moda son bienes aspiracionales. Estas características son la base del análisis de los productos de moda, de la definición de políticas comerciales y de los planes de marketing (Martínez Caballero y Vázquez Casco, 2006). Al respecto, el marketing de la moda es una función que permite a las empresas determinar qué productos se van a vender, a qué precios, cómo se van a comunicar y cuáles serán sus canales de distribución para competir en el mercado de la moda. En otras palabras, consiste en evaluar y seleccionar las oportunidades del mercado de la moda y establecer estrategias en un sector caracterizado por la innovación, las tendencias cambiantes y la competencia creciente.

El estudio de las tendencias es un aspecto fundamental en el mercado de la moda. El Diccionario de la Real Academia Española define al término tendencia como "una idea artística, económica, política, religiosa, que se orienta en una determinada dirección”. Por otra parte, Saulquin (1999) señala que "dado un conjunto de datos, recogidos durante un determinado período de tiempo, se llama tendencia a un tipo de evolución que se destaca por una línea que ajusta y da sentido a dicho conjunto". En el caso de la moda, la tendencia es la dirección o rumbo del mercado. Por lo tanto, las empresas de la moda deben investigar hacia donde se orientan las tendencias para poder permanecer en mercados cambiantes y competitivos.

En el mercado de la moda, los productos que se comercializan en el mismo tienen particularidades que se desprenden de todos los aspectos desarrollados anteriormente.

$\mathrm{Al}$ respecto, Grünfeld (2005) señala que "si hablamos de un producto de moda, fundamentalmente en el rubro indumentaria, lo físico-material en sí mismo se entretejerá con el corazón del producto imaginario". A partir de lo señalado anteriormente y lo desarrollado por Wilensky (2006), podríamos considerar que un producto de moda es, a la vez, tres productos: (a) producto físico: es el bien real o material, (b) producto simbólico: esta representado en avisos publicitarios y (c) producto imaginario: representa la imagen en la mente del consumidor. En este sentido, Grünfeld (2005) propone el siguiente ejemplo referido a un vestido de mujer: (a) producto físico: el vestido está hecho de seda, (b) producto simbólico: "viví la tersura acariciando tu piel" y (c) producto imaginario: la mujer se siente envuelta en alas de mariposa.

Con respecto al producto físico, podríamos señalar que es el resultado de un proceso que comienza con la investigación del mercado: la calle, las tendencias de los centros internacionales de la moda, las culturas regionales, los gustos y las necesidades de los clientes. Luego, pasamos a la fase del diseño que puede pertenecer a un diseñador famoso, un diseñador empleado de una empresa o un joven diseñador free lance. Seguidamente, comienza la fase de desarrollo de un producto en la cual pueden presentarse las alternativas de alta costura o de producto masivo y popular; entre ambas hay gran abanico de posibilidades. A continuación se pasa por las pruebas de concepto y de producto en las que se estudia la variedad de colores y, también, combinaciones a desarrollar y se realiza la serie de moldes de diferentes talles lo que implica definir cual es la edad, el cuerpo y el estilo del mercado meta. Con el correr del tiempo, el producto físico evolucionó tecnológicamente y esto tuvo relación con la evolución social. Por ejemplo, la fibra sintética sin plancha (wash and weare) se puso de moda a mediados del siglo XX debido a que la mujer comenzó a trabajar fuera de la casa. Esto pone en evidencia que "la moda representa un cambio social que refleja los cambios continuos que se producen en una época” (Beaton, 1990). Los productos de moda, al igual que cualquier producto, tienen un ciclo de vida. El modelo del ciclo de vida de un producto fue desarrollado por Levitt (1965). Según este autor, los produc- 
tos, como los seres vivos, nacen, crecen, se desarrollan y mueren.

Según Sandhusen (2002), el ciclo de vida del producto es un modelo que supone que los productos que se introducen a los mercados competitivos atraviesan un ciclo predecible con el transcurso del tiempo, el cual consta de una serie de fases. Cada una de las fases plantea amenazas y oportunidades que los especialistas de marketing deben tener en cuenta para mantener la rentabilidad del producto (Kotler y Keller, 2006, Lambin, 1995).

La curva del ciclo de vida de un producto puede presentar diversas formas, pero generalmente se la grafica con forma de campana. Esta curva se divide en distintas fases y cada una de ellas describe diferentes características, objetivos y estrategias (Kotler y Keller, 2006).

En el siguiente gráfico se indican las distintas fases del ciclo de vida de un producto de moda (Grünfeld, 1997):

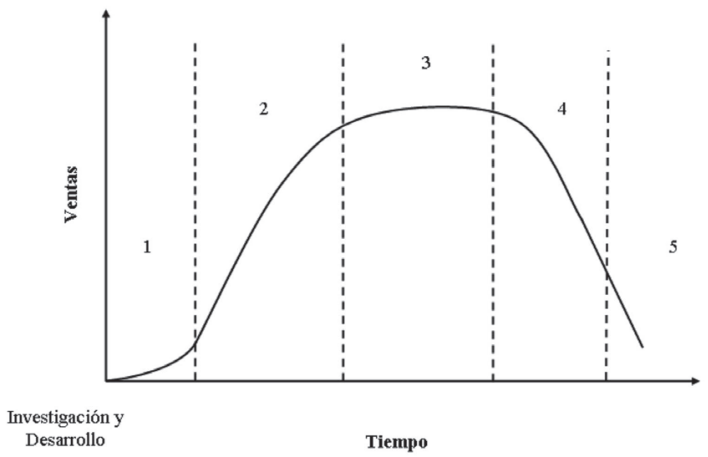

Figura 1. Ciclo de vida de un producto de moda. Fuente. Grünfeld, 1997.

\section{Introducción:}

En esta fase el producto se introduce en el mercado. Las características principales de esta etapa son las siguientes:

- Las ventas son bajas.

- El producto es adquirido solamente por un grupo reducido de personas denominado innovadores que son los primeros en adoptar una moda; son los líderes de opinión.

- La competencia es escasa o nula.

- El costo de lanzamiento es muy alto ya que no se ha cubierto la inversión que representa el diseño del producto, los desfiles, los catálogos para presentar las colecciones y otros costos.

- Los costos de realización son elevados por el escaso volumen producido.

- El precio es muy alto.

- La distribución es selectiva.

- La inversión en publicidad es alta.

- El riesgo es que el producto no sea aceptado. 


\section{Crecimiento:}

En esta etapa el producto es aceptado y cada vez más consumidores se interesan en ese estilo. Las características principales de esta fase son las siguientes:

- Las ventas aumentan fuertemente.

- El producto es adquirido por los "adoptadores tempranos" que siguen a los iniciadores y que quieren diferenciarse de la mayoría, pero no tienen la audacia o el poder económico de los primeros.

- La competencia es creciente.

- Los fabricantes copian los detalles más salientes y simplifican los restantes para poder responder a la demanda creciente. Realizan prácticamente los mismos modelos con telas y accesorios más económicos y con menos cuidado que los estilos originales.

- La producción en serie permite abaratar los precios y muchos consumidores pueden comprarlo.

- La distribución es intensiva.

- La inversión en publicidad es alta.

\section{Madurez o Culminación:}

Las características principales de esta fase son las siguientes:

- Las ventas se encuentran en el punto más alto y tienden a estabilizarse.

- El producto es adquirido por las "mayorías tempranas" que corresponden a grandes sectores que adoptan una moda porque les gusta, no se sienten incómodos al usarla porque la ven en los medios de difusión y en otras personas a quienes quieren parecerse.

- La competencia es intensa.

- El producto se vende y se distribuye mucho, hay numerosas variaciones dentro del estilo y los empresarios ofrecen diversos precios para ajustarse a las necesidades de cada consumidor, ya que se fabrican versiones caras y baratas dentro de la misma moda.

- Los precios son bajos.

- La distribución es intensiva.

- La inversión en publicidad es moderada.

- La duración del producto de moda en esta fase depende de su popularidad. Cuando logra convertirse en un clásico seguirá siendo consumido por varias temporadas y se ofrecerá con algún detalle que lo aggiorne o con algún cambio de telas o colores.

\section{Declinación:}

Las características principales de esta fase son las siguientes:

- Las ventas disminuyen.

- Las "mayorías tempranas" correspondientes a la fase anterior, comienzan a cansarse de los productos de esta moda porque observan que empiezan a venderse por debajo del precio que pagaron y se dan cuenta de su depreciación. Entonces, el producto es adquirido por las "mayorías tardías" que no estaban dispuestos a pagar el precio del producto cuando se encontraba 
en su apogeo. Estos clientes son más inseguros y compran los productos de moda cuando se han impuesto masivamente y se encuentran en todas las vidrieras de los locales.

- La distribución es selectiva.

- Los minoristas comienzan las liquidaciones porque necesitan pagar a sus proveedores y porque quieren tener lugar en el local para las colecciones nuevas. Lógicamente, mientras este producto de moda declina, otros productos están en las fases anteriores del ciclo de vida.

- La inversión publicitaria es mínima.

\section{Obsolescencia:}

En esta fase pocos consumidores quieren comprar el producto. Estos consumidores se denominan "rezagados" y adquieren el producto aunque ya haya pasado de moda.

Este es el momento en que se toma la decisión de discontinuar el producto y reemplazarlo por otro que se encuentre en la fase de iniciación del ciclo de vida.

El ciclo de vida descripto anteriormente corresponde a un producto de moda, pero existen otras dos categorías de ciclos de vida: estilos y modas pasajeras.

Como indicamos anteriormente, la moda es un estilo aceptado que goza de popularidad y aceptación en un campo dado y que tienen un carácter transitorio. En cambio, el estilo es una forma distintiva de expresión que aparece en un campo del quehacer humano. Por ejemplo, hay estilos en casas (colonial, gótico, renacentista, etc.); ropa (formal, casual, etc.); y arte (realista, surrealista, etc.). Un estilo puede perdurar en el tiempo y estar vigente por intervalos irregulares. Finalmente, las modas pasajeras aparecen, se adoptan rápidamente, alcanzan un máximo nivel en poco tiempo, y tienen una declinación igualmente rápida. Su ciclo de aceptación es corto, y tienden a atraer un número limitado de seguidores entre quienes están buscando emoción o quieren distinguirse de otros. Las modas pasajeras no sobreviven porque normalmente no satisfacen una necesidad intensa. Las mismas captan de inmediato el interés del público, son adoptadas con mucho entusiasmo, alcanzan pronto su nivel máximo y se esfuman rápidamente. Seguidamente, se graficarán las curvas referidas a estilo y moda pasajera (Kotler y Keller, 2006):

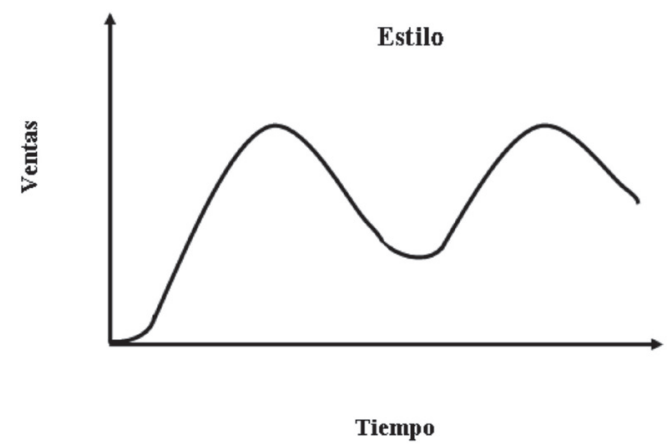

Figura 2. Estilo. Fuente. Kotler y Keller, 2006. 


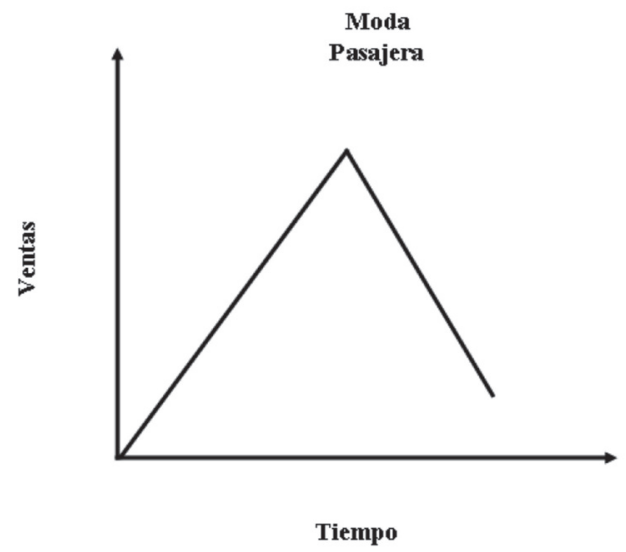

Figura 3. Moda pasajera. Fuente. Kotler y Keller, 2006.

Como hemos visto, el producto moda presenta un ciclo de vida compuesto por diferentes fases. $\mathrm{Al}$ respecto, Kotler y Keller (2006) señalan que la estrategia de diferenciación y posicionamiento debe cambiar a lo largo del ciclo de vida del producto. Los autores, consideran que aceptar que los productos tienen un ciclo de vida significa comprender que su duración será limitada. Además, implica admitir que las ventas de los mismos atravesarán diferentes fases y cada una de ellas presentará amenazas y oportunidades. Con respecto a las utilidades, las mismas aumentarán o disminuirán en las diferentes fases. Finalmente, los productos demandarán diferentes estrategias de marketing, de finanzas, de producción, de compras y de recursos humanos en cada una de las fases.

Conocer la fase del ciclo en la que se encuentra el producto de moda permitirá diseñar la estrategia más eficaz para prolongar su vida en el mercado. Dado que los tiempos turbulentos han venido para quedarse, este ejercicio de análisis y su constante práctica, rendirán sus frutos durante largos años.

\section{Referencias Bibliográficas}

Barthes, R. (1978). Sistema de la moda. Barcelona: Gustavo Gili.

Beaton, C. (1990). El espejo de la moda. Barcelona: Parsifal.

Grünfeld, M. (2005). Reflexiones sobre la moda. Trabajo Final en la Maestría "Generación y Análisis de Información Estadística”. Universidad de Tres de Febrero (UNTREF), Argentina. (1997). Marketing de la moda. Buenos Aires: Universo.

König, R. (1972). Sociología de la moda. Barcelona: Redondo.

Kotler, P. y Keller, K. L. (2006). Dirección de Marketing (12a ed.). México: Prentice Hall.

Lambin, J. (1995). Marketing estratégico (3a ed.). Madrid: McGraw-Hill.

Levitt, T. (1965). Exploit the product life cycle. Harvard Business Review, 43 (6), 81-94. 
Martínez Caballero, E., Vázquez Casco, A. (2006). Marketing de la moda. Madrid: Pirámide. Piñuel Raigada, J. L. (1992). La moda, o el aprendizaje de la integración por el cambio. Revista Española de Investigaciones Sociales (Rais), 57, 227-244.

Poiret P. (1930). En habillant l'epoque. París: Grasset.

Sandhusen, L. R. (2002). Mercadotecnia. México: Compañía Editorial Continental.

Saulquin, S. (1999). La moda, después. Buenos Aires: ISM.

(1998). La moda en la Argentina. Buenos Aires: Emecé.

Sproles, G. \& Burns, L. (1994). Changing Appearances: Understanding Dress in Contemporary

Society. New York: Fairchild Publications.

Sproles, G. (1985). Behavioral science theories of fashion. In Solomon, M. R. (Ed.), The psychology of fashion. Massachusetts: Lexington Books.

Wilensky, A. (2006). Marketing estratégico ( $8^{\mathrm{a}}$ ed.). Buenos Aires: Temas.

Summary: Fashion reflects the taste, the dominant values and views of a certain historical period. When people think about fashion, generally, imagine a fantasy world made up of designers, models and attractive clothing. Actually, fashion is not just a dress, but applies to any product or service consumer, for example, cars, music, furniture, magazines or tourist destinations.

If we consider fashion from the clothing point of view, we found that it has not only a sociocultural dimension found but also an economic one. Regarding the latter, we note that the fashion marketing functions is to determine which products will sell, to whom, at what prices, how they will communicate and what will be their distribution channels.

Often, it arises a confusion between fashion and style. Fashion refers to a product that becomes popular for a period of time and in a certain place. In style, this refers to the features and details that differentiate one product from others. Within the same style can be many variations in its creation: we are in presence of the designs. There are always many more design styles. When a style becomes popular becomes fashionable.

The products of fashion clothing, like any product, can apply the life cycle model. This model assumes that products are introduced to competitive markets go through a predictable cycle time. Each stage receives a specific name: introduction, growth, maturity and decline. Knowing the stage of the cycle which is the fashion product enables marketers to design the most effective strategy to market, which is particularly relevant in highly competitive markets in which the textile industry must act.

Keywords: clothing - design - fashion - life cycle - marketing - model - news - style.

Resumo: A moda reflete o gosto duma época, os valores e as opiniões dominantes. Quando as pessoas pensam na moda, geralmente, imaginam um mundo de fantasia conformado por designers, modelos e prendas atrativas. Em realidade, a moda não se refere somente à indumentária, senão que se aplica a qualquer produto ou serviço de consumo massivo como, por exemplo, automóveis, música, mobiliário, revistas, destinos turísticos, etc.

Se consideramos à moda desde o ponto de vista da indumentária, encontramos que tem uma 
dimensão sociocultural e, além, uma dimensão econômica. Em relação a esta última, podemos dizer que o marketing da moda tem como funções determinar que produtos se venderão, a quem, a que preço, como se vão comunicar e quais serão seus canais de comunicação.

Freqüentemente, as palavras moda e estilo se confundem. A moda se refere a todo produto que se converte em popular durante um período de tempo e num determinado lugar. $\mathrm{O}$ estilo se refere às características e os detalhes que diferenciam uns produtos de outros. Dentro de um mesmo estilo pode haver muitas variações em sua criação: estamos em presencia dos designs. Sempre há muitos mais designs que estilos. Quando um estilo se converte em popular se transforma em moda.

Aos produtos de moda do rubro indumentária, ao igual que qualquer produto, pode se the aplicar o modelo de ciclo de vida. Este modelo supõe que os produtos de se introduzem aos mercados competitivos atravessam um ciclo presumível no tempo. Cada etapa recebe um nome específico: introdução, crescimento, maturidade e declínio. Conhecer a etapa do ciclo na que se encontra o produto de moda, permite aos especialistas de marketing esboçar a estratégia mais eficaz para sua comercialização, aspecto relevante nos mercados altamente competitivos nos que a indústria têxtil deve atuar.

Palavras chave: ciclo de vida - design - estilo - indumentária - marketing - moda - modelo. 